\title{
COHEN-MACAULAY INVARIANT SUBALGEBRAS OF HOPF DENSE GALOIS EXTENSIONS
}

\author{
JI-WEI HE AND YINHUO ZHANG
}

\begin{abstract}
Let $H$ be a semisimple Hopf algebra, and let $R$ be a noetherian left $H$-module algebra. If $R / R^{H}$ is a right $H^{*}$-dense Galois extension, then the invariant subalgebra $R^{H}$ will inherit the AS-Cohen-Macaulay property from $R$ under some mild conditions, and $R$, when viewed as a right $R^{H}$-module, is a Cohen-Macaulay module. In particular, we show that if $R$ is a noetherian complete semilocal algebra which is AS-regular of global dimension 2 and $H=$ k $G$ for some finite subgroup $G \subseteq \operatorname{Aut}(R)$, then all the indecomposable CohenMacaulay module of $R^{H}$ is a direct summand of $R_{R^{H}}$, and hence $R^{H}$ is CohenMacaulay-finite, which generalizes a classical result for commutative rings. The main tool used in the paper is the extension groups of objects in the corresponding quotient categories.
\end{abstract}

\section{INTRODUCTION}

Motivated by the study of quotient singularities of noncommutative projective schemes, Van Oystaeyen and the authors introduced the concept of Hopf dense Galois extension in [HVZ2]. The present paper is a further study of Hopf dense Galois extensions. In this paper, we focus on the Cohen-Macaulay properties of the invariant subalgebra of a Hopf dense Galois extension. Let $H$ be a finite dimensional semisimple Hopf algebra, and let $R$ be a left $H$-module algebra. Let $H^{*}$ be the dual Hopf algebra. Since $H$ is finite dimensional, $R$ is a right $H^{*}$-comodule algebra. Denote by $R^{H}\left(=R^{c o H^{*}}\right)$ the invariant subalgebra. The algebra extension $R / R^{H}$ is called a right $H^{*}$-dense Galois extension ([HVZ2, Definition 1.1]) if the cokernel of the map

$$
\beta: R \otimes_{R^{H}} R \rightarrow R \otimes H^{*}, r \otimes r^{\prime} \mapsto(r \otimes 1) \rho\left(r^{\prime}\right)
$$

is finite dimensional, where $\rho: R \rightarrow R \otimes H^{*}$ is the right $H^{*}$-comodule structure map.

The concept of a Hopf dense Galois extension is a weaker version of that of a Hopf Galois extension. The main feature of a Hopf dense Galois extension is that there is an equivalence between some quotient categories. More precisely, let $R$ be a noetheiran algebra which is a left $H$-module algebra over a finite dimensional semisimple Hopf algebra $H$. Let $\operatorname{Mod} R$ be the category of right $R$-modules and let Tor $R$ be the full subcategory of $\operatorname{Mod} R$ consisting of torsion $R$-modules (see Section 1 ). Then we obtain a quotient category $\mathrm{QMod} R=\frac{\operatorname{Mod} R}{\operatorname{Tor} R}$. Set $B=R \# H$. Since $R$ is noetherian, $B$ and the invariant subalgebra $R^{H}$ are also noetherian. Then we also have quotient category QMod $B$ and QMod $R^{H}$. If $R / R^{H}$ is right $H^{*}$-dense Galois extension, then there is an equivalence of abelian categories QMod $R^{H} \cong \mathrm{QMod} B$ (cf. [HVZ2, Theorem 2.4]). Under this equivalence, we show in this paper that the

2000 Mathematics Subject Classification. Primary 16D90, 16E65, Secondary 16B50. 
invariant subalgebra $R^{H}$ inherits many homological properties from $R$. Especially, we have the following results (Theorems 3.5 and 4.5). The terminologies may be found in Section 3.

Theorem. Let $H$ be a finite dimensional semisimple Hopf algebra, and let $R$ be a noetherian left $H$-module algebra. Assume that the $H$-module $R$ is admissible and Tor $B$ is stable. If $R / R^{H}$ is a right $H^{*}$-dense Galois extension, then the following statements hold.

(i) If $R$ is $A S$-Cohen-Macaulay of dimension $d$, so is $R^{H}$.

(ii) If $R$ is $A S$-Cohen-Macaulay of dimension d, then $R$, viewed as a right $R^{H}$ module, is Cohen-Macaulay.

There are plenty of $H$-module algebras satisfying the assumptions in the theorem above. For example, if $R$ is a noetherian complete semilocal algebra and $H$ is a finite group algebra, then the $H$-module algebra $R$ is admissible and Tor $B$ is stable (cf. Example 2.5).

The main tool for proving of the theorem above is the following observation (Theorem 2.11) which gives a way to compute the extension groups of objects in the quotient category QMod $R^{H}$.

Theorem. Assume that the $H$-module algebra $R$ is admissible and Tor $B$ is stable. Let $N$ and $M$ be finitely generated right $B$-modules. Let $\mathcal{N}$ and $\mathcal{M}$ be the corresponding objects of $N$ and $M$ in the quotient categories. Then we have

(i) for each $i \geq 0, \operatorname{Ext}_{\mathrm{QMod} R}^{i}(\mathcal{N}, \mathcal{M})$ is a right $H$-module,

(ii) there are isomorphisms $\operatorname{Ext}_{\mathrm{QMod} B}^{i}(\mathcal{N}, \mathcal{M}) \cong \operatorname{Ext}_{\mathrm{QMod} R}^{i}(\mathcal{N}, \mathcal{M})^{H}, \forall i \geq 0$.

If $R$ is further a noetherian complete semilocal algebra which is also AS-regular of global dimension 2 , and $G \subseteq \operatorname{Aut}(R)$ is a finite subgroup, then the invariant subalgebra $R^{G}$ is Cohen-Macaulay-finite. Indeed, we have the following result (Theorem 5.3 ), which is a noncommutative version of a classical result for commutative rings due to Auslander $[\mathrm{A}]$.

Theorem. Let $R$ be a noetherian complete semilocal algebra, and let $G \subseteq \operatorname{Aut}(R)$ be a finite subgroup. Assume char $\mathbf{k} \nmid|G|$ and $R / R^{G}$ is a right $\mathbf{k} G^{*}$-dense Galois extension and $R$ is AS-regular of global dimension 2. Then the following statements hold.

(i) $R^{G}$ is AS-Cohen-Macaulay of dimension 2.

(ii) A finitely generated right $R^{G}$-module $M$ is Cohen-Macaulay if and only if $M \in \operatorname{add}\left(R_{R^{G}}\right)$, where $\operatorname{add}\left(R_{R^{G}}\right)$ is the subcategory of $\operatorname{Mod} R^{G}$ consisting of all the direct summands of finite direct sums of $R_{R^{G}}$.

When the algebra $R$ is graded, the above result was obtained by several authors (see Remark 5.5). However, the methods applied in graded case can not be applied to nongraded case directly. Our method depends on the relations of extension groups of objects in the quotient categories over $R^{G}$ and $R \# \mathbf{k} G$ respectively, which is different from the methods applies in the graded case.

Throughout, $\mathbf{k}$ is a field. All the algebras and modules are over $\mathbf{k}$. Unadorned $\otimes$ means $\otimes_{\mathbf{k}}$. We refer to [HVZ2] for basic properties of Hopf dense Galois extensions. 


\section{Hom-SETS OF THE QUOTIENT CATEGory}

Let $B$ be a (right) noetherian algebra. We denote by $\operatorname{Mod} B$ the category of right $B$-modules. Let $M$ be a right $B$-module. We say that a submodule $K$ of $M$ is cofinite if $M / K$ is finite dimensional. For an element $m \in M$, we say that $m$ is an torsion element if $m B$ is finite dimensional. Let $\Gamma_{B}(M)=\{m \in$ $M \mid m$ is a torsion element $\}$. Then $\Gamma_{B}(M)$ is a submodule of $M$. Note that $\Gamma_{B}$ is in fact a functor from $\operatorname{Mod} B$ to itself. The functor $\Gamma_{B}$ can be represented as $\Gamma_{B}=\lim \operatorname{Hom}_{B}(B / K,-)$, where $K$ runs over all the cofinite right ideals of $B$. In the sequel, if there is no risk of confusion, we will omit the subscript in the functor $\Gamma_{B}$, and simply write as $\Gamma$.

For a right $B$-module, if $\Gamma(M)=0$, then we say that $M$ is torsion free. If $\Gamma(M)=$ $M$, then $M$ is called a torsion module. Let Tor $B$ be the full subcategory of Mod $B$ consisting of torsion modules. One sees that Tor $B$ is a localizing subcategory of $\operatorname{Mod} B$. Hence we have an abelian quotient category $Q \operatorname{Mod} B:=\frac{\operatorname{Mod} B}{\operatorname{Tor} B}$. The natural projection functor is denoted by $\pi: \operatorname{Mod} B \rightarrow \mathrm{QMod} B$, which has a right adjoint functor $\omega:$ QMod $B \rightarrow \operatorname{Mod} B$. For $N, M \in \operatorname{Mod} B$, we write $\mathcal{N}$ and $\mathcal{M}$ for $\pi(N)$ and $\pi(M)$ respectively in the quotient category. The morphisms in QMod $B$ is defined to be the set

$$
\operatorname{Hom}_{M o d} B(\mathcal{N}, \mathcal{M})=\underset{\longrightarrow}{\lim } \operatorname{Hom}_{B}\left(N^{\prime}, M / \Gamma(M)\right),
$$

where $N^{\prime}$ runs over all the cofinite submodules of $N$, and the direct system is induced by the inclusion maps of the cofinite submodules.

If $M$ is a torsion free module, then its injective envelope is torsion free as well. The injective envelope of a torsion module is not torsion in general. If Tor $B$ is closed under taking injective envelope, then we say that Tor $B$ is stable. For example, if $B$ is a noetherian commutative local algebra, then $B$ has a stable torsion class.

Lemma 1.1. Let $N$ be a finitely generated right $B$-module, and let $E$ be an injective torsion B-module. Then $\lim \operatorname{Hom}_{B}\left(N^{\prime}, E\right)=0$, where $N^{\prime}$ runs over all the cofinite submodule of $N$.

Proof. For every cofinite submodule $N^{\prime}$ of $N$, applying the functor $\operatorname{Hom}_{B}(-, E)$ to the exact sequence $0 \rightarrow N^{\prime} \rightarrow N \rightarrow N / N^{\prime} \rightarrow 0$, we obtain the exact sequence $0 \rightarrow \operatorname{Hom}_{B}\left(N / N^{\prime}, E\right) \rightarrow \operatorname{Hom}_{B}(N, E) \rightarrow \operatorname{Hom}_{B}\left(N^{\prime}, E\right) \rightarrow 0$. Take the direct limit over all the cofinite submodules $N^{\prime} \subseteq N$, we obtain exact sequence

$$
0 \rightarrow \underset{\longrightarrow}{\lim } \operatorname{Hom}_{B}\left(N / N^{\prime}, E\right) \rightarrow \operatorname{Hom}_{B}(N, E) \rightarrow \underset{\longrightarrow}{\lim } \operatorname{Hom}_{B}\left(N^{\prime}, E\right) \rightarrow 0 .
$$

For each $B$-module morphism $f: N \rightarrow E$, since $N$ is finitely generated, $\operatorname{im} f$ is finite dimensional. Hence ker $f$ is cofinite. Since $f$ factors through $N / \operatorname{ker} f$, we see that the morphism $\lim _{\operatorname{Hom}_{B}}\left(N / N^{\prime}, E\right) \rightarrow \operatorname{Hom}_{B}(N, E)$ is an epimorphism, and hence $\lim _{\longrightarrow} \operatorname{Hom}_{B}\left(N^{\prime}, \vec{E}\right)=0$.

Lemma 1.2. Let $B$ be a noetherian algebra. Assume that Tor $B$ is stable. For every finitely generated module $N, M \in \operatorname{Mod} B$, if $N$ is finitely generated, then

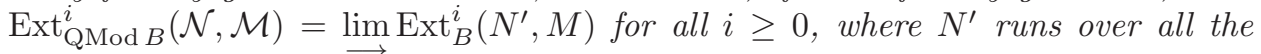
cofinite submodules of $\vec{N}$.

Proof. Take a minimal injective resolution of $M$ as follows

$$
0 \rightarrow M \rightarrow I^{0} \rightarrow I^{1} \rightarrow \cdots \rightarrow I^{n} \rightarrow \cdots
$$


Since Tor $B$ is stable, for each $n \geq 0, I^{n}=F^{n} \oplus E^{n}$ where $F^{n}$ is a torsion free module and $E^{n}$ is a torsion module. Note that if $F$ is an injective torsion free module, then $\mathcal{F}=\pi(F)$ is an injective object in QMod $B$. Applying the projection functor to the exact sequence (1.2.1), we obtain an injective resolution of $\mathcal{M}$ in QMod $B$ :

$$
0 \rightarrow \mathcal{M} \rightarrow \mathcal{F}^{0} \rightarrow \mathcal{F}^{1} \rightarrow \cdots \rightarrow \mathcal{F}^{n} \rightarrow \cdots
$$

Applying the functor $\operatorname{Hom}_{\mathrm{QMod} B}(\mathcal{N},-)$ to $(1.2 .2)$, we have

$$
0 \rightarrow \operatorname{Hom}_{\mathrm{QMod} B}\left(\mathcal{N}, \mathcal{F}^{0}\right) \rightarrow \cdots \rightarrow \operatorname{Hom}_{\mathrm{QMod} B}\left(\mathcal{N}, \mathcal{F}^{n}\right) \rightarrow \cdots,
$$

which is equivalent to the following complex

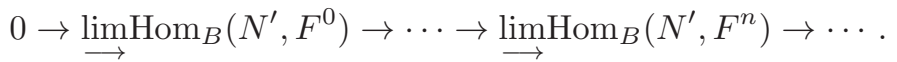

By Lemma 1.1, the complex (1.2.3) is equivalent to

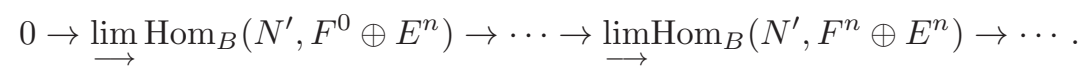

Taking the cohomology of (1.2.4), we obtain the desired result since the direct limit is exact.

\section{Hom-Sets of The QUOTIENT CATEgORIES OF SMASH PRODUCTS}

Throughout this section, let $R$ be a noetherian algebra, $H$ a finite dimensional semisimple Hopf algebra acting on $R$ so that $R$ is a left $H$-module algebra. Let $B=R \# H$ be the smash product. We may view $R$ as a left $B$-module by setting the left $B$-action

$$
(a \# h) \cdot r=a(h \cdot r), \forall a, r \in R, h \in H,
$$

where $h \cdot r$ is the left $H$-action on $R$. Similarly, we may view $R$ as a right $B$-module by setting the right $B$-action

$$
r \cdot(a \# h)=\left(S^{-1} h_{(1)} \cdot r\right)\left(h_{(2)} \cdot a\right), \forall a, r \in R, h \in H,
$$

where $S$ is the antipode of $H$ and $\Delta(h)=h_{(1)} \otimes h_{(2)}$.

Let $M$ be a right $B$-module. For every element $x \in M$, we see that $x B$ is finite dimensional if and only if $x R$ is finite dimensional since $H$ is finite dimensional. Hence we see as a right $B$-module $\Gamma_{R}(M)=\Gamma_{B}(M)$. From this observation, we obtain the following fact.

Lemma 2.1. Let $M$ be a right $B$-module. Then as right $R$-modules, $R^{i} \Gamma_{R}(M) \cong$ $R^{i} \Gamma_{B}(M)$ for all $i \geq 0$.

Proof. Let $0 \rightarrow M \rightarrow I^{0} \rightarrow I^{2} \rightarrow \cdots$ be an injective resolution of $M$ in the category of right $B$-modules. By [HVZ1, Proposition 2.6], each $I^{i}$ is injective as a right $R$ module. Hence $R^{i} \Gamma_{R}(M)$ is the $i$ th cohomology of the complex $\Gamma_{R}\left(I^{*}\right)$. Since $\Gamma_{R}\left(I^{i}\right)=\Gamma_{B}(M)$ for all $i \geq 0$, as complexes of right $R$-modules, $\Gamma_{R}\left(I^{*}\right)=\Gamma_{B}\left(I^{*}\right)$. Thus we have $R^{i} \Gamma_{R}(M)=R^{i} \Gamma_{B}(M)$.

Let $N$ be a right $R$-module. We define a right $B$-module $N \# H=N \otimes H$ whose right $B$-module action is given by $(n \otimes h)(a \otimes g)=n\left(h_{(1)} a\right) \otimes h_{(2)} g$ for $n \in N, g, h \in H$. 
Lemma 2.2. The following statements hold.

(i) If Tor $B$ is stable, then so is Tor $R$.

(ii) Let $A$ and $A^{\prime}$ be two noetherian algebras. If $F: \operatorname{Mod} A \longrightarrow \operatorname{Mod} A^{\prime}$ is an equivalence of abelian categories, then the restriction of $F$ to Tor $A$ induces an equivalence Tor $A \longrightarrow$ Tor $A^{\prime}$.

Proof. (i) Let $N$ be a torsion $R$-module. Set $M=N \# H$. Then $M$ is $B$-torsion module. Let $I$ be the injective envelope of $M$. Since Tor $B$ is stable, $I$ is a torsion $B$-module. By [HVZ1, Proposition 2.6], $I$ is injective as a right $R$-module. We view $N$ as an $R$-submodule of $M$ through the inclusion map $N \rightarrow M: n \mapsto n \# 1$. Then $N$ is a submodule of the torsion injective module $I$. Hence the injective envelope of $N$ must be torsion.

(ii) This is classical.

Proposition 2.3. Let $H$ be a finite dimensional semisimple and cosemisimple Hopf algebra. Let $R$ be a noetherian left $H$-module algebra. Then Tor $R$ is stable if and only if Tor $B$ is stable.

Proof. We only need to prove the "only if" part. Note that $B=R \# H$ is a right $H$-comodule algebra. It is a left $H^{*}$-module algebra. The invariant subalgebra $B^{H^{*}}=R$. Since $B$ is a right $H$-Galois extension of $R, R$ and $B \# H^{*}$ are Morita equivalent (cf. [CFM, Theorem 1.2]). Since Tor $R$ is stable, Tor $B \# H^{*}$ is stable by Lemma 2.2(ii). By Lemma 2.2(i), Tor $B$ is stable.

Let $N$ and $M$ be right $B$-modules. Note that $\operatorname{Hom}_{R}(N, M)$ has a right $H$-module action defined as follows: for $f \in \operatorname{Hom}_{R}(N, M), h \in H$,

$$
\left(f\llcorner h)(n)=f\left(n S\left(h_{(1)}\right)\right) h_{(2)}, \quad \forall n \in N .\right.
$$

Under this $H$-action on $\operatorname{Hom}_{R}(N, M)$, we have the following isomorphism [CFM]

$$
\operatorname{Hom}_{B}(N, M) \cong \operatorname{Hom}_{R}(N, M)^{H} \text {. }
$$

The right $H$-action on $\operatorname{Hom}_{R}(N, M)$ may be extended to the extension groups by choosing a projective resolution of $N$ (or injective resolution of $M$ ) in $\operatorname{Mod} B$. Moreover, we have the following isomorphisms [HVZ1]

$$
\operatorname{Ext}_{B}^{i}(N, M) \cong \operatorname{Ext}_{R}^{i}(N, M)^{H}, \forall i \geq 0 .
$$

Definition 2.4. We say that the left $H$-module algebra $R$ is (right) admissible if for any finitely generated right $B$-module $N$, and any cofinite $R$-submodule $K$ of $N$, there is a cofinite $B$-submodule $K^{\prime}$ of $N$ such that $K^{\prime} \subseteq K$.

There are several natural classes of noetherian admissible noetherian $H$-module algebras.

Example 2.5. Let $R$ be a noetherian semilocal algebra, that is, $R / J$ is a finite dimensional semisimple algebra where $J$ is the Jacobson radical of $R$. Let $G$ be a finite group which acts on $R$ so that $R$ is a left $G$-module algebra. Let $B=R \# \mathbf{k} G$. We know that $J$ is stable under the $G$-action. Let $N$ be a finitely generated right $B$-module, and let $K$ be an $R$-submodule such that $\bar{N}:=N / K$ is finite dimensional. Assume $\bar{N} \neq 0$. Note that $\bar{N} J \neq \bar{N}$. Since $\bar{N}$ is finite dimensional, there is an integer $k$ such that $\bar{N} J^{k}=0$. Hence $N J^{k} \subseteq K$. For $x \in N, r \in J^{k}$ and $g \in G$, we have $(x r) g=(x g)\left(g^{-1}(r)\right) \in N J^{k}$. Hence $N J^{k}$ is a $B$-submodule of $N$. Since 
$N /\left(N J^{k}\right)$ is a finitely generated module over $R / J^{k}$, it is finite dimensional. Hence the $G$-module algebra $R$ is admissible.

If $R$ is also complete with respect to the $J$-adic topology and char $\mathbf{k} \nmid|G|$, then Tor $B$ is stable. Indeed, a right $B$-module $M$ is torsion if and only if $M$ is torsion as a right $R$-module. Since $R$ is complete, by [Ja, Theorem 1.1], $J$ has the AtinRees property which insures that the injective envelope of a torsion $R$-module is still torsion. Since $\mathbf{k} G$ is both semisimple and cosemisimple, Tor $B$ is stable by Proposition 2.3.

Remark 2.6. In Definition 2.4, if $R$ is a graded algebra and the $H$-action is homogeneous, then we say that the left graded $H$-module algebra $R$ is admissible if the above conditions hold for finitely generated graded modules.

Example 2.7. Let $R=R_{0} \oplus R_{1} \oplus \cdots$ be a graded noetherian algebra such that $\operatorname{dim} R_{i}<\infty$ for all $i \geq 0$. Let $H$ be a finite dimensional semisimple Hopf algebra which acts on $R$ homogeneously so that $R$ is an $H$-module algebra. Set $B=R \# H$. Let $N$ be a graded finitely generated right $B$-module, and $K$ a graded $R$-submodule of $N$ such that $N / K$ is finite dimensional. Let $\mathfrak{m}=\oplus_{i \geq 1} R_{i}$. Since $N / K$ is finite dimensional, there is an integer $k$ such that $(N / K) \mathfrak{m}^{k}=0$. Hence $N \mathfrak{m}^{k} \subseteq K$. Since $\mathfrak{m}$ is stable under the $H$-action, $N \mathfrak{m}^{k}$ is a $B$-submodule of $N$. As $R$ is noetherian, $R / \mathfrak{m}^{k}$ is finite dimensional for all $k \geq 0$. Hence $N /\left(N \mathfrak{m}^{k}\right)$ is finite dimensional. It follows that the left $H$-module algebra $R$ is admissible in the graded sense.

Lemma 2.8. Let $R$ be an admissible $H$-module algebra. Let $N$ be a finitely generated right $B$-module, and $M$ a right $R$-module. Then

$$
\operatorname{Hom}_{\mathrm{QMod} R}(\mathcal{N}, \mathcal{M})=\underset{\longrightarrow}{\lim } \operatorname{Hom}_{R}(K, M),
$$

where $K$ runs over all the cofinite $B$-submodules of $N$.

Proof. By Lemma 1.2, $\operatorname{Hom}_{\mathrm{QMod} R}(\mathcal{N}, \mathcal{M})=\lim \operatorname{Hom}_{R}(K, M)$ where the limit runs over all the cofinite $R$-submodules $K$ of $\vec{N}$. Since the $H$-module algebra $R$ is assumed to be admissible, the direct system formed by all the cofinite $R$ submodules of $N$ and the direct system formed by all the cofinite $B$-submodules of $N$ are cofinal. Hence $\lim _{\longrightarrow} \operatorname{Hom}_{R}(K, M)=\lim _{\longrightarrow} \operatorname{Hom}_{R}\left(K^{\prime}, M\right)$, where on the left hand the direct limit runs over all the cofinite $\vec{R}$-submodules $K$ of $N$, and on the right hand the direct limit runs over all the cofinite $B$-submodules $K^{\prime}$ of $N$.

Proposition 2.9. Let $R$ be an admissible $H$-module algebra. Let $N$ and $M$ be finitely generated right $B$-modules. Then there is a natural right $H$-module action on $\operatorname{Hom}_{\mathrm{QMod}} R(\mathcal{N}, \mathcal{M})$. Moreover, with this $H$-module structure we have

$$
\operatorname{Hom}_{\mathrm{QMod} B}(\mathcal{N}, \mathcal{M}) \cong \operatorname{Hom}_{\mathrm{QMod} R}(\mathcal{N}, \mathcal{M})^{H},
$$

where the isomorphism is functorial in $M$.

Proof. By Lemma 2.8, $\operatorname{Hom}_{\mathrm{QMod} R}(\mathcal{N}, \mathcal{M})=\lim _{\longrightarrow} \operatorname{Hom}_{R}(K, M)$, where each $K$ in the direct system is a $B$-submodule of $N$. By $(2.3 .1), \operatorname{Hom}_{R}(K, M)$ is a right $H$ module for each cofinite $B$-submodule $K$. It is easy to see that the direct system is compatible with the right $H$-module actions. Hence $\operatorname{Hom}_{\mathrm{QMod} R}(\mathcal{N}, \mathcal{M})$ is a right $H$-module.

For each $B$-submodule $K$ of $N$, we have $\operatorname{Hom}_{B}(K, M) \cong \operatorname{Hom}_{R}(K, M)^{H}$ by (2.3.2). Since $H$ is semisimple, the functor ()$^{H}$ commutates with taking direct 
limits. Hence

$$
\begin{aligned}
\operatorname{Hom}_{\mathrm{QMod} B}(\mathcal{N}, \mathcal{M}) & =\underset{\lim }{\longrightarrow} \operatorname{Hom}_{B}(K, M) \\
& \cong \underset{\lim }{\longrightarrow} \operatorname{Hom}_{R}(K, M)^{H} \\
& \cong \stackrel{\left(\lim _{\longrightarrow} \operatorname{Hom}_{R}(K, M)\right)^{H}}{ } \\
& \cong \operatorname{Hom}_{\mathrm{QMod} R}(\mathcal{N}, \mathcal{M})^{H}
\end{aligned}
$$

If $M^{\prime}$ is another $B$-module and there is a right $B$-module morphism $f: M \rightarrow M^{\prime}$, then it is easy to see that the direct systems and the right $H$-module structures on $\operatorname{Hom}_{R}(K, M)$ and $\operatorname{Hom}_{R}\left(K, M^{\prime}\right)$ are compatible with the morphism $f$. Hence the isomorphism is functorial in $M$.

Next we show that the proposition above can be extended to extension groups, that is, there are natural $H$-module actions on extension groups $\operatorname{Ext}_{\mathrm{QMod} R}^{i}(\mathcal{N}, \mathcal{M})$ if the left $H$-module $R$ is admissible and Tor $B$ is stable.

For the rest of this section, the $H$-module algebra $R$ is admissible and Tor $B$ is stable. Let $M$ be a finitely generated right $B$-module. Take a minimal injective resolution of $M$ as follows:

$$
0 \rightarrow M \rightarrow I^{0} \rightarrow I^{1} \rightarrow \cdots \rightarrow I^{k} \rightarrow \cdots .
$$

Since Tor $B$ is stable, the injective envelope of a torsion module is still torsion. Then each injective module in the above sequence has a decomposition $I^{i}=E^{i} \oplus T^{i}$ such that $E^{i}$ is a torsion free $B$-module and $T^{i}$ is a torsion $B$-module. Write $I$ for the complex obtained from (2.9.1) by dropping $M$ on the left in the beginning. Similar to the discussions in [AZ, Section 7, P.271], we see that there are complexes $E^{*}, T^{\cdot}$ and a morphism $f: E^{\cdot}[-1] \rightarrow T^{*}$ such that $I^{\cdot}=$ cone $(f)$. Applying the projection functor $\pi: \operatorname{Mod} B \rightarrow$ QMod $B$ to the resolution (2.9.1) and noticing that $E^{i}$ is torsion free injective for all $i \geq 0$, we obtain an injective resolution of $\mathcal{M}$ in QMod $B$ :

$$
0 \rightarrow \mathcal{M} \rightarrow \mathcal{E}^{0} \rightarrow \mathcal{E}^{1} \rightarrow \cdots \rightarrow \mathcal{E}^{i} \rightarrow \cdots
$$

Note that any right $B$-module is also a right $R$-module. Moreover, a right $B$-module $K$ is torsion free if and only if it is torsion free as an $R$-module. We see that $E^{i}$ is also torsion free when it is viewed as an $R$-module, and $T^{i}$ is torsion as an $R$-module for all $i \geq 0$. By [HVZ1, Proposition 2.6], $E^{i}$ is also injective as a right $R$-module for each $i \geq 0$. Hence we have the following observation.

Lemma 2.10. Assume that Tor $B$ is stable. The sequence (2.9.2) is an injective resolution of $\mathcal{M}$ in $\mathrm{QMod} B$. If $\mathcal{M}$ and $\mathcal{E}^{i}(i \geq 0)$ are viewed as objects in QMod $R$ (by an abuse of the notions), then (2.9.2) is also an injective resolution of $\mathcal{M}$ in QMod $R$.

Theorem 2.11. Assume that $R$ is an admissible $H$-module algebra and Tor $B$ is stable. Let $N$ and $M$ be finitely generated right $B$-modules. Then for each $i \geq 0, \operatorname{Ext}_{\mathrm{QMod} R}^{i}(\mathcal{N}, \mathcal{M})$ is a right $H$-module, moreover, we have the following isomorphisms

$$
\operatorname{Ext}_{\mathrm{QMod} B}^{i}(\mathcal{N}, \mathcal{M}) \cong \operatorname{Ext}_{\mathrm{QMod} R}^{i}(\mathcal{N}, \mathcal{M})^{H}, \forall i \geq 0
$$


Proof. By Lemma 2.10, the sequence (2.9.2) is an injective resolution of $\mathcal{M}$ when viewed as an object in $\mathrm{QMod} R$. Applying $\operatorname{Hom}_{\mathrm{QMod} R}(\mathcal{N},-)$ to $(2.9 .2)$, we obtain

$$
0 \rightarrow \operatorname{Hom}_{\mathrm{QMod}}\left(\mathcal{N}, \mathcal{E}^{0}\right) \rightarrow \operatorname{Hom}_{\mathrm{QMod}}\left(\mathcal{N}, \mathcal{E}^{1}\right) \rightarrow \cdots \rightarrow \operatorname{Hom}_{\mathrm{QMod}}\left(\mathcal{N}, \mathcal{E}^{i}\right) \rightarrow \cdots .
$$

By Proposition 2.9, each component of the complex above is a right $H$-module and the differential is compatible with the right $H$-module structures. Taking the cohomology of the complex above, we obtain that $\operatorname{Ext}_{\mathrm{QMod} R}^{i}(\mathcal{N}, \mathcal{M})$ is a right $H$-module for each $i \geq 0$.

Now applying $\operatorname{Hom}_{\mathrm{QMod}} B(\mathcal{N},-)$ to the sequence $(2.9 .2)$, we obtain the following complex

(2.11.1) $\quad 0 \rightarrow \operatorname{Hom}_{\mathrm{QMod} B}\left(\mathcal{N}, \mathcal{E}^{0}\right) \rightarrow \operatorname{Hom}_{\mathrm{QMod} B}\left(\mathcal{N}, \mathcal{E}^{1}\right) \rightarrow \cdots \rightarrow \operatorname{Hom}_{\mathrm{QMod} B}\left(\mathcal{N}, \mathcal{E}^{i}\right) \rightarrow \cdots$

By Proposition 2.9, the complex (2.11.1) is isomorphic to the following complex

(2.11.2) $\quad 0 \rightarrow \operatorname{Hom}_{\mathrm{QMod} R}\left(\mathcal{N}, \mathcal{E}^{0}\right)^{H} \rightarrow \operatorname{Hom}_{\mathrm{QMod} R}\left(\mathcal{N}, \mathcal{E}^{1}\right)^{H} \rightarrow \cdots \rightarrow \operatorname{Hom}_{\mathrm{QMod} R}\left(\mathcal{N}, \mathcal{E}^{i}\right)^{H} \rightarrow \cdots$

By Lemma $2.10,(2.9 .2)$ is also an injective resolution of $\mathcal{M}$ in QMod $R$. Noticing that the functor ()$^{H}$ commutes with taking the cohomology, we obtain that the $i$ th cohomology of the complex (2.11.2) is equal to $\operatorname{Ext}_{\mathrm{QMod} R}^{i}(\mathcal{N}, \mathcal{M})^{H}$, which is isomorphic to $\operatorname{Ext}_{\mathrm{QMod} B}^{i}(\mathcal{N}, \mathcal{M})$, the $i$ th cohomology of (2.11.1) since (2.9.2) is also an injective resolution of $\mathcal{M}$ in $\mathrm{QMod} B$. Then we obtain the desired isomorphisms.

\section{Invariant subalgebras of Hopf Dense Galois extensions}

Throughout this section, $H$ is a finite dimensional semisimple Hopf algebra and $R$ is a noetherian $H$-module algebra. As before, set $B:=R \# H$ and $A=R^{H}$.

Setup 3.1. We assume that the $H$-module algebra $R$ satisfies the following conditions:

(i) the $H$-module algebra $R$ is admissible and Tor $B$ is stable (e.g. $R$ is a complete semilocal algebra, cf. Example 2.5);

(ii) $R / A$ is a right $H^{*}$-dense Galois extension.

As we have known in the beginning of Section $2, R$ is a $B$ - $A$-bimodule. Then we have a functor

$$
-\otimes_{B} R: \operatorname{Mod} B \longrightarrow \operatorname{Mod} A,
$$

which induces a functor between the corresponding quotient categories:

$$
-\otimes_{\mathcal{B}} \mathcal{R}: \operatorname{QMod} B \longrightarrow \mathrm{QMod} A \text {. }
$$

We recall a result obtained in [HVZ1].

Lemma 3.2. [HVZ1, Theorem 2.4] Assume that Setup 3.1(ii) holds. Then the functor

$$
-\otimes_{\mathcal{B}} \mathcal{R}: \operatorname{QMod} B \longrightarrow \operatorname{QMod} A
$$

is an equivalence of abelian categories.

Let $S$ be a noetherian algebra. Recall from Section 1 that we have a torsion functor $\Gamma_{S}: \operatorname{Mod} S \rightarrow \operatorname{Mod} S$. The $i$-th right derived functor of $\Gamma_{S}$ is denoted by $R^{i} \Gamma_{S}$. If there is no risk of confusion, we will drop the subscript $S$. Note that we have $\Gamma=\lim \operatorname{Hom}_{S}(R / K,-)$, and $R^{i} \Gamma=\lim \operatorname{Ext}_{S}^{i}(R / K,-)$, where $K$ runs over all the cofinite $S$-submodules of $S$, and the $\overrightarrow{\text { direct }}$ system is induced by the inclusion maps of cofinite submodules. 
Definition 3.3. We say that a noetherian algebra $S$ is (right) $A S$-Cohen-Macaulay of dimension $d$ if $R^{i} \Gamma(S)=0$ for all $i \neq d$.

Assume $S$ is AS-Cohen-Macaulay of dimension $d$. A finitely generated right $S$-module $M$ is said to be a Cohen-Macaulay module, if $R^{i} \Gamma(M)=0$ for all $i \neq d$

The definition of an AS-Cohen-Macaulay module is a generalization of the commutative version (cf. [CH, Z]). The letters "AS" stand for Artin-Schelter, because the property in the definition is also a generalization of that of Artin-Schelter Gorenstein algebras (cf. [VdB, Remark 8.5]).

Lemma 3.4. Let $S$ be a noetherian algebra such that Tor $S$ is stable. For a finitely generated right $S$-module, we have

(i) $\operatorname{Ext}_{\mathrm{QMod} S}^{i}(\mathcal{S}, \mathcal{M}) \cong R^{i+1} \Gamma(M)$ for $i>0$, and

(ii) an exact sequence $0 \rightarrow \Gamma(M) \rightarrow M \stackrel{\phi}{\rightarrow} \operatorname{Hom}_{\mathrm{QMod} S}(\mathcal{S}, \mathcal{M}) \rightarrow R^{1} \Gamma(M) \rightarrow 0$, where $\phi$ is the natural map $M=\operatorname{Hom}_{S}(S, M) \stackrel{\pi}{\rightarrow} \operatorname{Hom}_{\mathrm{QMod} S}(\mathcal{S}, \mathcal{M})$ induced by the projection functor $\pi: \operatorname{Mod} S \rightarrow$ QMod $S$.

Proof. For any cofinite right $S$-submodule $K$ of $S$, we have an exact sequence $0 \rightarrow K \rightarrow S \rightarrow S / K \rightarrow 0$, which implies isomorphisms

$$
\operatorname{Ext}_{S}^{i}(K, M) \cong \operatorname{Ext}_{S}^{i+1}(S / K, M), \forall i \geq 1
$$

and an exact sequence

$$
0 \rightarrow \operatorname{Hom}_{S}(S / K, M) \rightarrow M \stackrel{\phi}{\rightarrow} \operatorname{Hom}_{S}(K, M) \rightarrow \operatorname{Ext}_{S}^{1}(S / K, M) \rightarrow 0,
$$

where $\phi$ is the natural map induced by the inclusion map $K \hookrightarrow S$. Taking direct limit on all the cofinite submodules of $S$, by Lemma 1.2 and $R^{i} \Gamma(M)=$ $\lim \operatorname{Ext}_{S}^{i}(S / K,-)$ for all $i \geq 0$, we obtain the desired results.

Theorem 3.5. Assume that $R$ and $H$ satisfy the conditions in Setup 3.1. If $R$ is $A S$-Cohen-Macaulay of dimension d, then so is $R^{H}$.

Proof. Set $A:=R^{H}$. By Lemma 3.2, the functor $-\otimes_{\mathcal{B}} \mathcal{R}:$ QMod $B \longrightarrow \operatorname{QMod} A$ is an equivalence of abelian categories. Under this equivalence, the object $\mathcal{R} \in$ QMod $B$ corresponds to $\mathcal{A} \in \operatorname{QMod} A$. Then we have

$$
\operatorname{Ext}_{\mathrm{QMod} A}^{i}(\mathcal{A}, \mathcal{A}) \cong \operatorname{Ext}_{\mathrm{QMod} B}^{i}(\mathcal{R}, \mathcal{R}), \forall i \geq 0 \text {. }
$$

Combining (3.5.1) with Theorem 2.11, we obtain

$$
\operatorname{Ext}_{\mathrm{QMod} A}^{i}(\mathcal{A}, \mathcal{A}) \cong \operatorname{Ext}_{\mathrm{QMod} R}^{i}(\mathcal{R}, \mathcal{R})^{H}, \forall i \geq 0 .
$$

Assume $d \geq 2$. Then $\operatorname{Ext}_{\mathrm{QMod} R}^{i}(\mathcal{R}, \mathcal{R})=0$ for all $i \neq d-1$ and $i \geq 1$ by Lemma 3.4(i), and hence $\operatorname{Ext}_{\mathrm{QMod} A}^{i}(\mathcal{A}, \mathcal{A})=0$ for all $i \neq d-1$ and $i \geq 1$. By Lemma 3.4(i) again, $R^{i+1} \Gamma_{A}(A)=0$ for $i \neq d-1$ and $i \geq 1$. Since $R^{i} \Gamma_{R}(R)=0$ for $i \neq d$, Lemma 3.4(ii) implies that the natural map $R \rightarrow \operatorname{Hom}_{\mathrm{QMod} R}(\mathcal{R}, \mathcal{R})$ is an isomorphism. Applying the functor ()$^{H}$ on this isomorphism, we see that the natural map $A \rightarrow \operatorname{Hom}_{\mathrm{QMod} A}(\mathcal{A}, \mathcal{A})$ is an isomorphism. Hence $R^{i} \Gamma_{A}(A)=0$ for $i=0,1$.

Now assume $d=1$. Similar to the above discussions, we have $R^{i} \Gamma_{A}(A)=0$ for $i \geq 2$. Since $\Gamma_{R}(R)=0$, Lemma 3.4(ii) implies that the natural map $R \stackrel{\phi}{\rightarrow}$ $\operatorname{Hom}_{\mathrm{QMod} R}(\mathcal{R}, \mathcal{R})$ is injective. Applying the functor ()$^{H}$ to this map, we obtain that the natural map $A \stackrel{\phi}{\rightarrow} \operatorname{Hom}_{\mathrm{QMod}} A(\mathcal{A}, \mathcal{A})$ is injective. Hence $\Gamma_{A}(A)=0$. The case that $d=0$ can be proved similarly. 


\section{Cohen-Macaulay property of $R$ AS An $R^{H}$-Module}

Keep the same notions as in Section 3. We recall some properties of modules over a Hopf algebra.

Let $X$ be a right $H$-module. The tensor product $X \otimes H$ has two right $H$-module structures. The first one is the diagonal action of $H$, that is, $(x \otimes h) \cdot g=x \cdot g_{(1)} \otimes h g_{(2)}$ for $x \in X, g, h \in H$. To avoid possible confusion, the tensor product $X \otimes H$ with the diagonal action of $H$ will be denoted by $X \hat{\otimes} H$. The other $H$-action is the multiplication of elements of $H$ on the right of $X \otimes H$, that is, $(x \otimes h) \cdot g=x \otimes h g$ for $x \in X$ and $g, h \in H$. This right $H$-module structure will be denoted by $X \otimes H_{\bullet}$. The following lemma is well known.

Lemma 4.1. Let $X$ be a right $H$-module. Define maps

$$
\varphi: x \hat{\otimes} H \longrightarrow M \otimes H_{\bullet}, x \otimes h \mapsto x S\left(h_{(1)}\right) \otimes h_{(2)},
$$

and

$$
\psi: X \otimes H_{\bullet} \longrightarrow X \hat{\otimes} H, x \otimes h \mapsto x h_{(1)} \otimes h_{(2)} .
$$

Then $\varphi$ and $\psi$ are $H$-module isomorphisms which are inverse to each other.

Let $X$ be a right $H$-module. Recall from the beginning of Section 2 that $R$ is a right $B$-module by the right $B$-action (2.0.2). Then $\operatorname{Hom}_{R}(X, R)$ is a right $H$-module. Note that $B$ itself is a right $B$-module. Hence $\operatorname{Hom}_{R}(X, B)$ is a right $H$-module.

Lemma 4.2. Let $X$ be a right $B$-module. Then we have an isomorphism of right $H$-modules

$$
\operatorname{Hom}_{R}(X, R) \hat{\otimes} H \cong \operatorname{Hom}_{R}(X, R \# H) .
$$

Proof. Firstly, note that the smash product may be written as $H \# R$ with the multiplication defined by

$$
(h \# r)\left(g \# r^{\prime}\right)=h g_{(2)} \#\left(S^{-1} g_{(1)} r\right) r^{\prime} .
$$

There is an isomorphism of algebras:

$$
\xi: H \# R \rightarrow R \# H, h \# r \mapsto h_{(1)} r \# h_{(2)} .
$$

Define a linear map

$$
\zeta: \operatorname{Hom}_{R}(X, R) \hat{\otimes} H \longrightarrow \operatorname{Hom}(X, R \# H)
$$

by $\zeta(f \otimes h)(x)=h_{(1)} f(x) \# h_{(2)}$ for $f \in \operatorname{Hom}_{R}(X, R), x \in X$ and $h \in H$. We first show that $\zeta(f \otimes h)$ is a right $R$-module morphism for every $f \in \operatorname{Hom}_{R}(X, R)$ and $h \in H$. For $r \in R$ and $x \in X$, we have

$$
\begin{aligned}
\zeta(f \otimes h)(x r) & =h_{(1)} f(x r) \# h_{(2)} \\
& =h_{(1)}(f(x) r) \# h_{(2)} \\
& =\left(h_{(1)} f(x)\right)\left(h_{(2)} r\right) \# h_{(3)} \\
& =\left(h_{(1)} f(x) \# h_{(2)}\right) r \\
& =\zeta(f \otimes h)(x) r .
\end{aligned}
$$

Hence the image of $\zeta$ is indeed in $\operatorname{Hom}_{R}(X, R \# H)$. Therefore we obtain a map (still use the same notion):

$$
\zeta: \operatorname{Hom}_{R}(X, R) \hat{\otimes} H \longrightarrow \operatorname{Hom}_{R}(X, R \# H) .
$$


We next check that $\zeta$ is a right $H$-module morphism. For $f \in \operatorname{Hom}_{R}(X, R), h, g \in$ $H$ and $x \in X$, we have

$$
\begin{aligned}
\zeta(f \otimes h) \leftarrow g(x) & =\left(\zeta(f \otimes g)\left(x S\left(g_{(1)}\right)\right) g_{(2)}\right. \\
& =\left(h_{(1)} f\left(x S\left(g_{(1)}\right)\right) \# h_{(2)}\right) g_{(2)} \\
& =h_{(1)} f\left(x S\left(g_{(1)}\right)\right) \# h_{(2)} g_{(2)} .
\end{aligned}
$$

On the other hand, we have

$$
\begin{aligned}
\zeta((f \otimes h) \cdot g)(x) & =\zeta\left(f \leftarrow g_{(1)} \otimes h g_{(2)}\right)(x) \\
& =h_{(1)} g_{(2)} f<g_{(1)}(x) \# h_{(2)} g_{(3)} \\
& =h_{(1)} g_{(3)} S^{-1}\left(g_{(2)}\right) f\left(x S\left(g_{(1)}\right)\right) \# h_{(2)} g_{(4)} \\
& =h_{(1)} f\left(x S\left(g_{(1)}\right) \# h_{(2)} g_{(2)},\right.
\end{aligned}
$$

where the third equality follows from the definitions of the right $B$-module action (2.0.2) on $R$ and the right $H$-action on $\operatorname{Hom}_{R}(X, R)$. Therefore, $\zeta$ is a right $H$ module morphism.

Since $H \# R$ is a finitely generated free $R$-module, it follows that

$$
\theta: \operatorname{Hom}_{R}(X, R) \otimes H \longrightarrow \operatorname{Hom}_{R}(X, H \# R), f \otimes h \mapsto[x \mapsto h \otimes f(x)]
$$

is a linear isomorphism. Thus $\zeta$, equal to $\operatorname{Hom}_{R}(X, \xi) \circ \theta$, is an isomorphism since $\xi$ is an isomorphism.

Lemma 4.3. Let $X$ be a right $B$-module. For each $i \geq 0$, we have an $H$-module isomorphism

$$
\operatorname{Ext}_{R}^{i}(X, R \# H) \cong \operatorname{Ext}_{R}^{i}(X, R) \hat{\otimes} H .
$$

Moreover, the isomorphism is functorial in $X$.

Proof. Take a projective resolution of the right $B$-module $X$ as follows:

$$
\cdots \longrightarrow P^{i} \longrightarrow \cdots \longrightarrow P^{1} \longrightarrow P^{0} \longrightarrow X \longrightarrow 0 .
$$

By [HVZ1, Proposition 2.5], each $P^{i}$ is also projective as a right $R$-module. Apply the functor $\operatorname{Hom}_{R}(-, B)$ on (4.3.1) to obtain the following complex

$$
0 \longrightarrow \operatorname{Hom}_{R}\left(P^{0}, B\right) \longrightarrow \operatorname{Hom}_{R}\left(P^{1}, B\right) \longrightarrow \cdots \longrightarrow \operatorname{Hom}_{R}\left(P^{i}, B\right) \longrightarrow \cdots
$$

By Lemma 4.2, the complex (4.3.2) can be written in the following way (4.3.3)

$$
0 \longrightarrow \operatorname{Hom}_{R}\left(P^{0}, R\right) \hat{\otimes} H \longrightarrow \operatorname{Hom}_{R}\left(P^{1}, R\right) \hat{\otimes} H \longrightarrow \cdots \longrightarrow \operatorname{Hom}_{R}\left(P^{i}, R\right) \hat{\otimes} H \longrightarrow \cdots
$$

Comparing the cohomology of (4.3.2) and (4.3.3), we obtain the desired isomorphisms. It follows from the general homology theory that the isomorphism is functorial in $X$.

Proposition 4.4. Let $N$ be a finitely generated right B-module. Assume that the $H$-module algebra $R$ is admissible and Tor $B$ is stable. For each $i \geq 0$, we have

$$
\operatorname{Ext}_{\text {QMod } B}^{i}(\mathcal{N}, \mathcal{B}) \cong \operatorname{Ext}_{\text {QMod } R}^{i}(\mathcal{N}, \mathcal{R}) .
$$

Proof. By Lemma 1.2 and the assumption that the $H$-module algebra $R$ is admissible, $\operatorname{Ext}_{\mathrm{QMod} R}^{i}(\mathcal{N}, \mathcal{B}) \cong \underset{\longrightarrow}{\longrightarrow} \operatorname{Ext}_{R}^{i}\left(N^{\prime}, B\right)$ where $N^{\prime}$ runs over all the cofinite $B$-submodules of $N$. By Lemma 4.3, $\operatorname{Ext}_{R}^{i}\left(N^{\prime}, B\right) \cong \operatorname{Ext}_{R}^{i}\left(N^{\prime}, R\right) \hat{\otimes} H$ for all cofinite 
$B$-submodules $N^{\prime}$ of $N$. Taking the direct limits over all the cofinite $B$-submodules of $N$, we obtain

$$
\operatorname{Ext}_{\mathrm{QMod} R}^{i}(\mathcal{N}, \mathcal{B}) \cong \operatorname{Ext}_{\mathrm{QMod} R}^{i}(\mathcal{N}, \mathcal{R}) \hat{\otimes} H .
$$

Combining Theorem 2.11 and Lemma 4.1, we have

$$
\operatorname{Ext}_{\mathrm{QMod} B}^{i}(\mathcal{N}, \mathcal{B}) \cong\left(\operatorname{Ext}_{\mathrm{QMod} R}^{i}(\mathcal{N}, \mathcal{R}) \otimes H_{\bullet}\right)^{H} .
$$

The right hand side of (4.4.2) is isomorphic to $\operatorname{Ext}_{\mathrm{QMod} R}^{i}(\mathcal{N}, \mathcal{R})$ as a vector space.

Now we arrive at our main result of this section.

Theorem 4.5. Assume that $R$ and $H$ satisfy the conditions in Setup 3.1. If $R$ is AS-Cohen-Macaulay of dimension $d$, then $R$, viewed as a right $R^{H}$-module, is Cohen-Macaulay (cf. Definition 3.3).

Proof. Set $A:=R^{H}$. Since $R / A$ is a right $H^{*}$-dense Galois extension, the functor $-\otimes_{\mathcal{B}} \mathcal{R}:$ QMod $B \rightarrow$ QMod $A$ is an equivalence of abelian categories (cf. Lemma 3.2 ). Note that $R$ may be viewed both as a right $A$-module and as a right $B$ module. Under the equivalence $-\otimes_{\mathcal{B}} \mathcal{R}, \mathcal{R}$ when viewed as an object in QMod $A$ corresponds to $\mathcal{B} \in \mathrm{QMod} B$, and $\mathcal{A} \in \mathrm{QMod} A$ corresponds to $\mathcal{R}$ which is viewed as an object in QMod $B$. Hence for $i \geq 0$, we have

$$
\operatorname{Ext}_{\mathrm{QMod} B}^{i}(\mathcal{R}, \mathcal{B}) \cong \operatorname{Ext}_{\mathrm{QMod} A}^{i}(\mathcal{A}, \mathcal{R}) .
$$

By Proposition 4.4, we obtain

$$
\operatorname{Ext}_{\mathrm{QMod} R}^{i}(\mathcal{R}, \mathcal{R}) \cong \operatorname{Ext}_{\mathrm{QMod} A}^{i}(\mathcal{A}, \mathcal{R}) .
$$

The rest of the proof is almost the same as that of Theorem 3.5 according to the value of $d$. We omit the similar narratives.

Remark 4.6. Under the assumptions in Theorem 4.5, the isomorphism (4.5.1) implies that

$$
R^{i} \Gamma_{R^{H}}(R) \cong R^{i} \Gamma_{R}(R), \text { for all } i \geq 0 .
$$

If the algebra $R$ is a noetherian complete semilocal algebra and some further conditions are satisfied, then (4.6.1) follows from [WZ1, Theorem 2.9].

\section{Finite GRoup aCtions on NOETherian COMPlEte SEMILOCAL ALGEBRAS}

Recall that a noetherian algebra $\Lambda$ is semilocal if $\Lambda / J(\Lambda)$ is a finite dimensional semisimple algebra, where $J(\Lambda)$ is the Jacobson radical of $\Lambda$. We say that $\Lambda$ is complete if $\Lambda$ is complete with respect to the $J(\Lambda)$-adic topology, or equivalently, $\Lambda=\lim \Lambda / J(\Lambda)^{n}$.

Recall from [WZ2] that a noetherian semilocal algebra $\Lambda$ is called a right $A S$ Gorenstein algebra if $\Lambda_{\Lambda}$ has finite injective dimension $d$, $\operatorname{Ext}_{\Lambda}^{i}(\Lambda / J(\Lambda), \Lambda)=0$ for $i \neq d$, and $\operatorname{Ext}_{\Lambda}^{d}(\Lambda / J(\Lambda), \Lambda) \cong \Lambda / J(\Lambda)$ as a left $\Lambda$-module. Left AS-Gorenstein algebras are defined similarly. If $\Lambda$ is both left and right AS-Gorenstein, then we simply say that $\Lambda$ is $A S$-Gorenstein. If, furthermore, $\Lambda$ has finite global dimension, then we say that $\Lambda$ is $A S$-regular. We remark that our definition of AS-Gorenstein algebras is a little stronger than that in [WZ2]. 
Lemma 5.1. Let $\Lambda$ be a noetherian semilocal algebra. Then

$$
\Gamma_{\Lambda} \cong \lim _{n \rightarrow \infty} \operatorname{Hom}_{\Lambda}\left(\Lambda / J(\Lambda)^{n},-\right) .
$$

Proof. Recall from Section $1, \Gamma_{\Lambda}=\underset{\longrightarrow}{\lim _{\operatorname{Hom}}}(\Lambda / K,-)$ where $K$ runs over all the cofinite right ideal of $\Lambda$. For any cofinite right ideal $K$ of $\Lambda$, there is an integer $n$ such that $(\Lambda / K) J(\Lambda)^{n}=0$. Then $J(\Lambda)^{n} \subseteq K$. Hence the direct system defined by all the cofinite right ideals of $S$ and the direct system defined by $\left\{J(\Lambda)^{n} \mid n \geq 1\right\}$ are cofinal. Therefore, $\Gamma_{\Lambda}=\lim _{\longrightarrow} \operatorname{Hom}_{\Lambda}(\Lambda / K,-) \cong \lim _{n \rightarrow \infty} \operatorname{Hom}_{\Lambda}\left(\Lambda / J(\Lambda)^{n},-\right)$.

In the rest of this section, we assume $R$ that is a noetherian complete semilocal algebra with Jacobson radical $J$. Let $G \subseteq \operatorname{Aut}(R)$ be a finite subgroup. Then $R$ becomes a left $G$-module algebra. As before, set $B=R \# \mathbf{k} G$ and $A=R^{G}$. Example 2.5 shows that the $G$-module algebra $R$ is admissible and Tor $B$ is stable. Note that $J$ is stable under the $G$-action. Let $\mathfrak{m}=J \# \mathbf{k} G$. Then $\mathfrak{m}$ is a cofinite ideal of $B$, and $\mathfrak{m}^{n}=J^{n} \# \mathbf{k} G$ for all $n \geq 1$. In general, $\mathfrak{m}$ is not the Jacobson radical of $B$. Besides, for a general semisimple Hopf algebra $H$, an $H$-action on $R$ does not imply that the $H$ acts on the Jacobson radical $J$ stably. Some discussions on the stability of the $H$-action on the Jacobson radical, or on the Jacobson radical of the smash product may be found in [LMS].

Similar to the proof of Lemma 5.1, we also have

$$
\Gamma_{B} \cong \lim _{n \rightarrow \infty} \operatorname{Hom}_{B}\left(B / \mathfrak{m}^{n},-\right) .
$$

Recall that the depth of a right module $M$ over a noetherian algebra $\Lambda$ is defined as follows

$$
\operatorname{depth}_{\Lambda}(M)=\min \left\{i \mid R^{i} \Gamma_{\Lambda}(M) \neq 0\right\} .
$$

We have the following Auslander-Buchsbaum formula, which is a consequence of [WZ1, Theorem 0.1(1)]. We remark that our definition of the depth of a module is equivalent to that in [WZ1] when the algebra $\Lambda$ is a notherian complete semilocal algebra (see also, Lemma 5.4 below).

Lemma 5.2. Let $R$ be a noetherian complete semilocal algebra which is also ASGorenstein of injective dimension $d$. For a finitely generated right $B$-module $M$ with finite projective dimension, we have $\operatorname{projdim}_{B}(M)+\operatorname{depth}_{B}(M)=d$.

Proof. Note that $M$ is also a finitely generated right $R$-module. By Lemma 2.1, $\operatorname{depth}_{B}(M)=\operatorname{depth}_{R}(M)$. By [WZ1, Theorem 0.1(1)], we have

$$
\operatorname{projdim}_{R}(M)=d-\operatorname{depth}_{R}(M)=d-\operatorname{depth}_{B}(M) .
$$

Since a projective right $B$-module is projective as a right $R$-module, $\operatorname{projdim}_{R}(M) \leq$ $\operatorname{projdim}_{B}(M)$. On the other hand, set $p=\operatorname{projdim}_{R}(M)$. Let $\cdots \rightarrow P^{i} \stackrel{\delta^{i}}{\rightarrow} \cdots \stackrel{\delta^{1}}{\rightarrow}$ $P^{0} \rightarrow M \rightarrow 0$ be a projective resolution of the right $B$-module $M$. Then the $p$ th syzygy ker $\delta^{p-1}$ of the resolution is projective as an $R$-module since $\operatorname{projdim}_{R}(M)=$ $p$. By [HVZ1, Proposition 2.5], $\operatorname{ker} \delta^{p-1}$ is projective as a right $B$-module, and hence $\operatorname{projdim}_{B}(M) \leq p$. Therefore $\operatorname{projdim}_{R}(M)=\operatorname{projdim}_{B}(M)$. By the equalities (5.2.1), $\operatorname{projdim}_{B}(M)+\operatorname{depth}_{B}(M)=d$.

It is not hard to see that if a noetherian complete semilocal algebra is ASGorenstein with injective dimension $d$, then it is AS-Cohen-Macaulay of dimension $d$ in the sense of Definition 3.3 (cf. [WZ1]). 
Theorem 5.3. Let $R$ be a noetherian complete semilocal algebra, and let $G \subseteq$ $\operatorname{Aut}(R)$ be a finite subgroup. Assume that $R / R^{G}$ is a right $\mathbf{k} G^{*}$-dense Galois extension and that $R$ is AS-regular of global dimension 2. Set $A=R^{G}$. Then the following statements hold.

(i) A is AS-Cohen-Macaulay of dimension 2.

(ii) A finitely generated right $A$-module $M$ is Cohen-Macaulay if and only if $M \in \operatorname{add}\left(R_{A}\right)$, where $\operatorname{add}\left(R_{A}\right)$ is the subcategory of $\operatorname{Mod} A$ consisting of all the direct summands of finite direct sums of $R_{A}$.

Proof. The statement (i) is a special case of Theorem 3.5.

(ii) By Theorem 4.5, $R_{A}$ is a Cohen-Macaulay module. Hence any module in $\operatorname{add} R_{A}$ is Cohen-Macaulay.

Conversely, note that the functor $-\otimes_{\mathcal{B}} \mathcal{R}:$ QMod $B \rightarrow$ QMod $A$ in Lemma 3.2 is induced by the functor $-\otimes_{B} R: \operatorname{Mod} B \rightarrow \operatorname{Mod} A$ (cf. [HVZ2, Sections 2 and $3]$ ), that is, we have the following commutative diagram

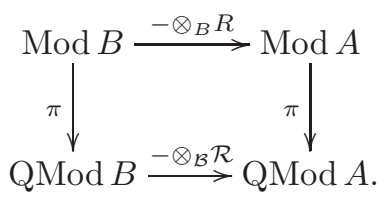

Let $F=-\otimes_{B} R$ and $\mathcal{F}=-\otimes_{\mathcal{B}} \mathcal{R}$. The functor $F$ has a right adjoint functor $F^{\prime}:=\operatorname{Hom}_{A^{\circ}}(R,-)$ (cf. [HVZ2, Section 2]), where $\operatorname{Hom}_{A^{\circ}}(-,-)$ is the Homfunctor in the category of left $A$-modules. Moreover, $F F^{\prime} \cong \operatorname{id}_{\operatorname{Mod} A}$. Hence $F^{\prime}$ is fully faithful and $\operatorname{Hom}_{B}\left(F^{\prime} X, F^{\prime} Y\right) \stackrel{F}{\longrightarrow} \operatorname{Hom}_{A}(X, Y)$ is an isomorphism for any $X, Y \in \operatorname{Mod} A$.

Let $N_{A}$ be a nonzero finitely generated Cohen-Macaulay module. Then we obtain that the map $\operatorname{Hom}_{B}\left(F^{\prime}(R), F^{\prime}(N)\right) \stackrel{F}{\longrightarrow} \operatorname{Hom}_{A}(R, N)$ is an isomorphism. Since $R / A$ is a right $\mathbf{k} G^{*}$-dense Galois extension and $R$ is AS-regular of global dimension 2, the natural map $R \# \mathbf{k} G \rightarrow \operatorname{Hom}_{A}(R, R), r \# h \mapsto\left[r^{\prime} \mapsto r\left(h \cdot r^{\prime}\right)\right]$ is an isomorphism of algebras by [HVZ2, Theorem 3.10]. Hence as a right $B$-module, $F^{\prime}(R)=\operatorname{Hom}_{A}(R, R)$ is isomorphic to $B$. Set $M=F^{\prime}(N)=N \otimes_{A} R$.

From the above commutative diagram (5.3.1), we obtain the following commutative diagram

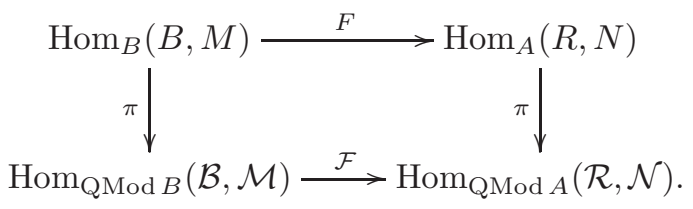

The top map in the diagram (5.3.2) is an isomorphism. By Lemma 3.2, the bottom map is also an isomorphism. Consider the exact sequence

$$
0 \rightarrow K \rightarrow R \rightarrow R / K \rightarrow 0,
$$

where $K$ is a cofinite submodule of $R$. Applying $\operatorname{Hom}_{A}(-, N)$ to the above sequence, we obtain the following exact sequence

$$
0 \rightarrow \operatorname{Hom}_{A}(R / K, N) \rightarrow \operatorname{Hom}_{A}(R, N) \rightarrow \operatorname{Hom}_{A}(K, N) \rightarrow \operatorname{Ext}_{A}^{1}(R / K, N)
$$

Since $N$ is Cohen-Macaulay and $R / K$ is a finite dimensional, $\operatorname{Hom}_{A}(R / K, N)=0$ and $\operatorname{Ext}_{A}^{1}(R / K, N)=0$ by Lemma 5.4 below. Hence (5.3.3) implies that the natural 
map

$$
\operatorname{Hom}_{A}(R, N) \rightarrow \operatorname{Hom}_{A}(K, N)
$$

is an isomorphism for all cofinite submodules $K$ of $R$.

Taking the direct limits on both sides of the isomorphism above over all the cofinite submodules $K$ of $R$, we obtain that the projection map

$$
\operatorname{Hom}_{A}(R, N) \stackrel{\pi}{\rightarrow} \operatorname{Hom}_{\mathrm{QMod} A}(\mathcal{R}, \mathcal{N})
$$

is an isomorphism.

In summary, we obtain that the top map, the bottom map and the right vertical map in the diagram (5.3.2) are all isomorphisms. Hence the left vertical map in the diagram (5.3.2) is also an isomorphism.

By Lemma 5.1, the exact sequence $0 \rightarrow \mathfrak{m}^{n} \rightarrow B \rightarrow B / \mathfrak{m} \rightarrow 0$ implies the next exact sequence

$$
0 \rightarrow \Gamma_{B}(M) \rightarrow \operatorname{Hom}_{B}(B, M) \stackrel{\pi}{\rightarrow} \operatorname{Hom}_{\mathrm{QMod} B}(\mathcal{B}, \mathcal{M}) \rightarrow R^{1} \Gamma_{B}(M) \rightarrow 0 .
$$

Since the projection map $\pi$ is an isomorphism, $\Gamma_{B}(M)=R^{1} \Gamma_{B}(M)=0$. Since $R$ is of global dimension 2, the global dimension of $B$ is 2 as well (cf. [L]). By Lemma $5.2, M$ is a projective $B$-module. Hence $M$ is a direct summand of a free module $B^{(n)}$. Therefore $N=F(M)$ is a direct summand of $F(B)^{(n)}=\left(R_{A}\right)^{(n)}$, that is, $N \in \operatorname{add}\left(R_{A}\right)$.

Lemma 5.4. Let $A$ be a noetherian algebra. Let $M_{S}$ be a finitely generated module. If $R^{i} \Gamma_{A}(M)=0$ for $i<d$, then $\operatorname{Ext}_{A}^{i}(T, M)=0$ for all finite dimensional right A-module $T$.

Proof. Take a minimal injective resolution of $M$ as follows

$$
0 \rightarrow M \rightarrow I^{0} \stackrel{\delta^{0}}{\rightarrow} I^{1} \stackrel{\delta^{1}}{\rightarrow} \cdots \stackrel{\delta^{i-1}}{\rightarrow} I^{i} \stackrel{\delta^{i}}{\rightarrow} \cdots .
$$

We claim that each $I^{i}$ is torsion free for all $i<d$. Since $\Gamma_{A}(M)=0$, $M$ is torsion free. Hence $I^{0}$ is torsion free. Now suppose that there is some $i<d$ such that $I^{i}$ is not torsion free. Assume that $k<d$ is the smallest integer such that $\Gamma_{A}\left(I^{k}\right) \neq 0$. Since the injective resolution is minimal, $\operatorname{ker} \delta^{k}$ is essential in $I^{k}$. Hence $\operatorname{ker} \delta^{k} \cap \Gamma_{A}\left(I^{k}\right) \neq 0$. Then the kernel of the restriction $\delta^{k}: \Gamma_{A}\left(I^{k}\right) \rightarrow \Gamma_{A}\left(I^{k+1}\right)$ is nontrivial. Therefore $R^{k} \Gamma_{A}(M) \neq 0$, a contradiction. Hence for each $i<d$, $I^{i}$ is torsion free. Therefore, for any finite dimensional module $T, \operatorname{Hom}_{A}\left(T, I^{i}\right)=0$ for all $i<d$, which implies that $\operatorname{Ext}_{A}^{i}(T, M)=0$.

Remark 5.5. The above theorem may be viewed as a nongraded version of [Jo, Section 3], [Ue1, Corollary 1.3] (see also [Ue2, Proposition 5.2]) and [CKWZ, Theorem 4.4]. Our method is also valid for noetherian graded algebras (cf. Example 2.7), however our method is quite different from those in [Jo, Ue1, CKWZ].

Acknowledgments. J.-W. He is supported by NSFC (No. 11571239, 11401001) and Y. Zhang by an FWO-grant. 


\section{REFERENCES}

[A] M. Auslander, Rational singularities and almost split sequences, Trans. Amer. Math. Soc. 293 (1986), 511-531.

[AZ] M. Artin, J. J. Zhang, Noncommutative projective schemes, Adv. Math. 109 (1994), 228287.

[CH] O. Celikbas and H. Holm, Equivalences from tilting theory and commutative algebra from the adjoint functor point of view, arXiv:1703.06685, 2017.

[CFM] M. Cohen and D. Fischman, S. Montgomery, Hopf Galois extensions, Smash products, and Morita equivalence, J. Algebra 133 (1990), 351-372.

[CKWZ] K. Chan, E. Kirkman, C. Walton, and J.J. Zhang, McKay Correspondence for semisimple Hopf actions on regular graded algebras, part II, arXiv:1610.01220, 2016.

[Ja] A. V. Jategaonkar, Morita duality and Noetherian rings, J. Algebra 69 (1981), 358-371.

[Jo] P. Jørgensen, Finite Cohen-Macaulay type and smooth non-commutative schemes, Canad. J. Math., 60 (2008), 379-390.

[HVZ1] J.-W. He, F. Van Oystaeyen and Y. Zhang, Hopf algebra actions on differential graded algebras and applications, Bull. Belg. Math. Soc. Simon Stevin 18 (2011), 99-111.

[HVZ2] J.-W. He, F. Van Oystaeyen and Y. Zhang, Hopf dense Galois extensions with applications, J. Algebra 476 (2017), 134-160.

[L] G. Liu, A note on global dimension of smash products, Comm. Algebra 33 (2005), 2625-2627.

[LMS] V. Linchenko, S. Montgomery, L. W. Small, Stable Jacobson radicals and semiprime smash products, Bull. London Math. Soc. 37 (2005), 860-872.

[VdB] M. Van den Bergh, Existence theorems for dualizing complexes over non-commutative graded and filtered rings, J. Algebra 195 (1997), 662-679.

[Ue1] K. Ueyama, Noncommutative graded algebra of finite Cohen-Macaulay representation type, Proc. Amer. Math. Soc. 143 (2015), 3703-3715.

[Ue2] K. Ueyama, Graded maximal Cohen-Macaulay modules over noncommutative Gorenstein isolated singularities, J. Algebra 383 (2013), 85-103.

[WZ1] Q.-S. Wu, J. J. Zhang, Dualizing complexes over noncommutative local rings, J. Algebra 239 (2001), 513-548.

[WZ2] Q.-S. Wu, J. J. Zhang, Homological identities for noncommutative rings, J. Algebra 242 (2001), 516-535.

[Z] M. R. Zargar, On the relative Cohen-Macaulay modules, J. Algebra Appl. 14 (2015), 1550042, $7 \mathrm{pp}$.

He: Department of Mathematics, Hangzhou Normal University, Hangzhou Zhejiang 310036, CHINA

E-mail address: jwhe@hznu.edu.cn

Zhang: Department of Mathematics and Statistics, University of Hasselt, Universitaire Campus, 3590 Diepenbeek, Belgium

E-mail address: yinhuo.zhang@uhasselt.be 